\title{
3 Research Square

\section{Willingness to use HIV pre-exposure prophylaxis and associated factors among men who have sex with men in Liuzhou, China}

\section{Yuansheng Fu}

Anhui Medical University

Akililu Alemu Ashuro

Anhui Medical University

\section{Xianxiang Feng}

Liuzhou Center for Disease Control and Prevention

\section{Ting Wang}

Anhui Medical University

Sichen Zhang

Anhui Medical University

Dongqing Ye

Anhui Medical University

Yin Guang Fan ( $\nabla$ fanyinguang@163.com )

Anhui Medical University

\section{Research}

Keywords: Pre-exposure prophylaxis, Men who have sex with men, HIV prevention, Willingness, China

Posted Date: March 23rd, 2021

DOl: https://doi.org/10.21203/rs.3.rs-336411/v1

License: (c) (i) This work is licensed under a Creative Commons Attribution 4.0 International License.

Read Full License

Version of Record: A version of this preprint was published at AIDS Research and Therapy on July 30th, 2021. See the published version at https://doi.org/10.1186/s12981-021-00374-8. 


\section{Abstract}

Background: HIV pre-exposure prophylaxis (PrEP) is a key component of HIV combination prevention strategies, which has the potential to decrease the incidence of HIV in men who have sex with men (MSM). This study aimed to evaluate the levels of PrEP acceptability and explore the factors associated with willingness to use PrEP among MSM in Liuzhou, China.

Methods: Between November 2017 and May 2019, a cross-sectional survey was conducted among 829 MSM. The study participant were recruited through nonprobability sampling method. Participants' demographics, HIV/AIDS knowledge, sexual behaviors, drug use and HIV test history were collected. Multivariate logistic regression was performed to identify factors associated with willingness to use PrEP.

Results: A total of 829 MSM completed the survey, and $30.28 \%(95 \% \mathrm{Cl}$ : $27.3-33.4)$ were willing to use PrEP. In multivariate logistic regression, factors associated with a higher willingness to use PrEP include, being Zhuang or Han ethnic origin, recruited through peer introduction or gay venues, pursing higher education level, previous HIV testing and having oral sex with a man. However, had a casual sex in the past six months were associated with lower willingness to use PrEP.

Conclusions: MSM in Liuzhou reported a relatively low level of willingness to use PrEP. The results indicate the need for effective education, targeted intervention, and define implementation strategies to promote PrEP acceptance among MSM.

\section{Background}

Human immunodeficiency virus (HIV) is the virus that causes acquired immune deficiency syndrome (AIDS) and has become a global public health burden, which results significant mortality and morbidity(1). In China, about 850,000 people were living with HIV in 2018(2). Men who have sex with men (MSM) were considered to be a key population at high risk of HIV infection. The HIV prevalence among MSM in China has increased significantly from $1.4 \%$ in 2005 to $8.0 \%$ in 2015(3). Additionally, MSM contributed over a quarter of new HIV diagnoses in China(4). Despite the availability of various HIV prevention methods, such as condom use, HIV and sexually transmitted infections (STIs) testing and antiretroviral therapy (ART), the HIV epidemic among MSM remained uncontrolled, especially in the western part of China $(5,6)$. In view of these trends, there is an urgent need for China to implement new and effective HIV prevention measures among MSM.

HIV pre-exposure prophylaxis (PrEP), a new biomedical approach for HIV prevention using antiretroviral drugs (tenofovir disoproxil fumarate /emtricitabine, TDF/FTC) before HIV exposure among HIV-negative individuals, is increasingly recognized as the most promising HIV prevention strategy(7). Clinical trials and cohort studies have demonstrated the safety and efficacy of oral PrEP in reducing the risk of HIV acquisition among MSM(8), heterosexual men and women(9) and people who inject drugs(10). In 2015, the World Health Organization (WHO) recommended the use of oral PrEP as an additional prevention choice to all people at substantial risk of HIV infection, including MSM(11). Currently, the United States, 
France, Brazil, South Africa and several other countries have approved the use of PrEP among MSM. In China, PrEP is still in research stage and has not yet been incorporated into a nation-wide HIV prevention program.

While PrEP is obviously effective, its success ultimately depends on the adoption of those who need it. A growing body of evidence from different time periods and locations has shown that MSM play an increasingly important role in China's HIV/AIDS epidemic(12). Recent national reports showed that HIV transmission among MSM accounted for $21.4-23.4 \%$ of the newly identified HIV/AIDS cases in some areas in China(13). In recent years, research on the willingness to use PrEP among MSM have been conducted in many countries (14-16). However, the prevalence of willingness varies across countries, which may limit the general application of existing studies. In addition, due to the socio-demographic, cultural, and structural factors which were likely to affect the acceptability of PrEP among MSM(4), it was important to understand how to implement PrEP according to local settings.

Liuzhou is a city with the highest prevalence of HIV infection in Guangxi Zhuang Autonomous Region which has the second-largest number of people living with HIV in south central China(17). And MSM ranked the second place among the number of newly identified HIV/AIDS cases in Liuzhou city(18). However, there were no available data on willingness to use PrEP and factors among MSM in this region. Therefore, the aim of this study is to assess the level of willingness to use PrEP and explore its relationship with socio-demographic factors, HIV/AIDS knowledge, sexual behaviors, drug use and HIV test history among MSM in Liuzhou. Findings on the willingness to use PrEP and associated factors could provide a basis and guidance for application and promotion in terms of PrEP implementation among MSM in Liuzhou, China.

\section{Methods}

\section{Participants and procedure}

A cross-sectional study was conducted among MSM persons who lived in Liuzhou, China from November 2017 to May 2019. Participants were recruited through a nonprobability sampling method and the main methods of recruitment included: (1) finding the "seed" subjects, through peer introduction and the "snowball" sampling method to find other research subjects; (2) via network platform (i.e., websites and social media applications); and (3) recruited by trained and experienced peer educators from spaces frequented by MSM, such as parks, bathhouses, and bars. The inclusion criteria were as follows: (1) aged 15 years or older; (2) born biologically male; (3) had anal and/or oral intercourse with at least one man in the last six months; (4) HIV-negative; and (5) residing in Liuzhou. HIV-positive persons were excluded and referred to the local Centers for Disease Control and Prevention (CDC). Eligible participants were briefed about the study's purpose and informed consent was obtained from each subject prior to enrolling each into the study. Each participant completed a face-to-face interview questionnaire in a private room, which took approximately 20 minutes to complete. Respondents were compensated 50 RMB (about 7.5 US dollars) for their time spent on the survey. A total of 854 participants were contacted, of whom 829 were 
eligible and completed the survey. The study protocol was approved by the ethics committee of Anhui Medical University.

\section{Laboratory tests}

Three milliliters of blood samples was collected and transported to the laboratory of local CDC within 12 hours. Plasma specimens were screened for HIV antibodies by using enzyme-linked immunosorbent assay (ELISA; Beijing Modern Gaoda Biotechnology Co., Beijing, China), and positive tests were confirmed by a HIV-1/2 Western blot assay (HIV Blot 2.2 WBH; Genelabs Diagnostics, Singapore).

\section{Measurement}

Demographic information collected include age, household registration, ethnicity, occupation, education level, marital status, personal monthly income, sexual orientation, history of sexually transmitted diseases (STDs) other than HIV, and recruitment source.

In all, HIV/AIDS knowledge was measured with six questions for which two questions were on mosquito bites and number of sex partners in HIV transmission, another two questions on condom use and lubricant use in HIV prevention, and one also on blood testing for HIV as well as one other question on HIV/AIDS treatment. There was one point assigned for a correct answer, 0 for a wrong answer or an answer of unknown or unsure. The total score for HIV literacy ranged from 0 to 6 , and a score equal to or greater than 4 was classified as "high level of HIV/AIDS knowledge" and scores below 4 were classified as "low level of HIV/AIDS knowledge".

Items for assessing sexual behaviors were made up of two scales (homosexual and heterosexual). The scale of homosexual behaviors was measured by the following questions: total number of male sexual partners, age at first anal sex with men, number of anal sex partners in the past month, condom use in anal sexual intercourse, oral sexual behavior with men, and commercial sex with men in the past six months. Heterosexual behaviors was evaluated by the following questions: total number of female sexual partners, age at first anal sex with women and heterosexual behavior in the past six months.

Participants were asked if they had ever taken any drugs (including ecstasy, heroin, marijuana, opium, crystal meth, methamphetamine, cocaine, sedatives or stimulants, etc.) and the frequency of taking the drugs in the past month. In addition, participants were also asked about their HIV testing history and whether they knew the test results.

To assess the willingness of PrEP, respondents were provided a brief description of PrEP (i.e., PrEP is a daily medication that people who do not have HIV take to prevent getting infected with HIV. PrEP is taken before someone is exposed to HIV.), and then asked them to select a response from a 5-point Likert scale $(1=$ definitely not, 2 = probably not, $3=$ not sure, 4 = probably will, 5 = definitely will). Responses were then dichotomized. Choices 1, 2, and 3 were classified into the "unwilling to use PrEP" group and choices 4 and 5 were classified into the "willing to use PrEP" group. 


\section{Statistical analysis}

All statistical analyses were performed using SPSS version 23.0 (SPSS Inc., Chicago, IL, USA). Descriptive statistics, including the frequency, percentage, mean and standard deviation (SD) were reported. Chisquare test and t-test were used to compare differences in demographic, HIV/AIDS knowledge, sexual behaviors, drug use and HIV testing history between MSM who were willing and those who unwilling to use PrEP. Variables with a p-value $<0.05$ in the univariate analysis were selected and further explored in a multivariable logistic regression model. Multivariate logistic regression analyses were performed to evaluate the factors associated with willingness to use PrEP. Odd ratios (ORs) and $95 \%$ confidence intervals $(95 \% \mathrm{Cl})$ were calculated. A two-tailed $p<0.05$ was considered as statistically significant.

\section{Results}

\section{Participants characteristics}

A total of 854 participants were enrolled in this study. All participants were tested for HIV screening and 25 of them were positive and excluded from the study. Thus, 829 eligible MSM were included in the final analysis. Among those eligible MSM, 251 (30.28\%, 95\% Cl: 27.3-33.4) reported that they were willing to use PrEP for HIV prevention.

The majority of participants were younger than 35 years old (81.2\%). The mean age was $28.5( \pm 9.0)$. More than half of participants were local residents (57.3\%), and $43.7 \%$ of participants were of Han ethnicity. Over one-fifth of respondents were freelancers and $69.6 \%$ had either college or higher level of education. The majority of participants reported their sexual orientation as homosexual (76.2\%), and $72.7 \%$ of participants were never married. In addition, $5.8 \%$ self-reported a history of STDs other than HIV. The sample source was mainly from peer introduction (37.9\%) and network recruitment (30.0\%) (Table 1).

\section{HIV/AIDS knowledge}

The mean score of HIV/AIDS knowledge scale was $3.0( \pm 0.92)$, ranging from 0 to 6 . Only $25.7 \%$ of the 829 MSM participants had a score of 4 or higher, while $74.3 \%$ scored below 4 points. There was no difference in willingness to use PrEP by HIV/AIDS knowledge $(\chi 2=0.369, P=0.544)($ Table 1$)$.

\section{Sexual behavior characteristics}

In terms of homosexual behaviors, $90.1 \%$ of participants reported having two or more male sex partners in their lifetime with $11.8 \%$ having 20 or more male sex partners. More than $90 \%$ of participants reported ever had anal intercourse with male sex partners, and the mean age of first anal sex with a man was 22.64 years old $( \pm 6.34)$. Around half $(46.8 \%)$ of participants had two or more male anal sex partners in the past month, and $27.5 \%$ using no condom for their last anal sex. $89.0 \%$ of participants reported ever had oral sex with a man and $44.6 \%$ had two or more male oral sex partners in the past month. Only a few participants (5.1\%) had commercial sex with a man in the last six months, whereas $40.4 \%$ reported that they have had a casual sex other than commercial sex in the past six months. Regarding heterosexual 
behaviors, about $45.0 \%$ reported having one or more female sex partners in their lifetime and the mean age of first sex with a woman was 22.15 years old $( \pm 3.90)$. More than half of participants $(56.6 \%)$ reported that they had not used condom in their last sex with a woman (Table 2).

\section{Drug use and HIV test history}

Overall, $12.2 \%$ (101/ 829) of participants reported that they had ever use drugs. The median time of drug use in the past month was one (IQR: 0-2). About one third of participants (32.1\%) had never tested for HIV. Among the 563 participants who had a history of HIV test, $95.2 \%$ (536/563) reported they knew the test results. Those who had a history of HIV test were more likely to be willing to use PrEP compared to those who did not $(X 2=21.356, P<0.001)$.

\section{Factors associated with willingness to use PrEP}

In the univariate analysis, ethnicity, education level, monthly income, sexual orientation, history of STDs, recruitment source, ever had oral sex with a man, had casual sex other than commercial sex in the past six months and history of HIV test were significantly associated with willingness to use PrEP.

All the variables associated with willingness to use PrEP in univariate analyses were included in a multivariate logistic regression model. The results of multivariate logistic regression model showed that comparing to other ethnic groups, Zhuang ( $\mathrm{aOR}=3.172,95 \% \mathrm{Cl}$ : $1.696,5.934)$ and Han ethnic origin (aOR $=8.785,95 \% \mathrm{Cl}: 4.722,16.343)$ were more likely to be willing to use PrEP. As compared to participants recruited through other ways, those recruited through peer introduction $(\mathrm{aOR}=3.584,95 \% \mathrm{Cl}: 2.104,6.106)$ and gay venues $(\mathrm{aOR}=22.940,95 \% \mathrm{Cl}: 11.608,45.053)$ were more likely to be willing to use PrEP. In addition, men who had a higher education level (college or above: aOR $=1.881,95 \% \mathrm{Cl}$ : 1.082, 3.268; reference group: middle school or below), ever had oral sex with a man (aOR $=1.949,95 \% \mathrm{Cl}: 1.013$, 3.749 ) and history of HIV test ( $\mathrm{aOR}=2.341,95 \% \mathrm{Cl}: 1.552,3.532)$ were also significantly and positively associated with willingness to use PrEP. However, had a casual sex in the past six months $(\mathrm{aOR}=0.381$, $95 \% \mathrm{Cl}: 0.261,0.557)$ was significantly and negatively associated with willingness to use PrEP. (Table 3 ).

\section{Discussion}

In this study, demographic characteristics, including Han and Zhuang ethnic origin and pursuing college or above education were related to willing to use PrEP. In addition, the sample source from the peer introduction and gay venues were related to willing to use PrEP. As well, a history of HIV test and having oral sex with a man were related to willingness to use PrEP. However, had a causal sex in the past six months was significantly and negatively associated with willingness to use PrEP.

In this finding, the proportion of MSM in willing to use PrEP was higher than study conducted in Shanghai (19) and it is lower than Chengdu's study (20). The possible explanation, which might be due to different sampling techniques. In our study, participants were included from several sampling sources that might cause a proportion difference with the other studies. Compared to studies from PrEP implemented 
settings, willingness to use PrEP was lower among MSM in Liuzhou, China. Studies from US revealed that, willingness to use PrEP has ranged from 46.1-61.0\% $(21,22)$. Similarly, our finding was also lower than studies conducted in Scotland and Spain $(23,24)$. Unsurprisingly, Liuzhou is an economically undeveloped city located in southwest China, and it might be difficult for the inhabitants to adapt to a new lifestyle. Therefore developing strategies that would motivate MSM to engage in novel HIV prevention.

Moreover, this study showed that MSM who graduated from college or above have shown higher willingness to use PrEP. This finding suggested that higher education have significant impact on willing to use PrEP. Previous study had revealed that participants with higher education were more likely to have better knowledge of STDs and its prevention (25). Similarly, MSM who have a higher education might have better access to have health related information. In Brazil, MSM who reported that learning about PrEP online positively influenced their willingness to use PrEP (26). In addition, this finding highlighted the need to increase access to PrEP related knowledge among less educated.

In this study, we found that participants who recruited through peer introduction were more willing to use PrEP. Peers were more likely than health care workers to influence the behaviors of fellow group members. It had been reported that peer education was an effective method that increased HIV related knowledge (27). A meta-analysis revealed that peer-led interventions increased HIV testing among MSM (28). Linked in peer group might encourage MSM to discuss about HIV prevention strategies with their friends. In Berlin, Germany, $90 \%$ of MSM reported already of being aware of PrEP in which $61.7 \%$ of MSM got knowledge from their friends or acquaintances (29). Similarly, recent study revealed that PrEP willingness among MSM were related to previous PrEP awareness (24). Our finding support recommendation for using peer group as potentially effective medium for dissemination of PrEP messaging to be rollout in entire China.

Gay venue mostly used for sourcing male sex partners by MSM and it was a place where high-risk behaviors occurred. In China, the HIV incidence is growing faster among MSM, especially those who frequently visited gay venues (30). We found that participants who recruited from gay venues were more likely to be willing to use PrEP. In London, men who used gay venues frequently were more likely to report impact on STDs knowledge than those who didn't use (31). The association of gay venues and PrEP willingness might be related to a higher risk perception among those who visited more frequently, as well as to a higher exposure to prevention messages from gay communities during meeting venues.

Furthermore, in this finding, ever had oral sex with a man was associated with willingness to use PrEP. The association of having oral sex and PrEP willingness might be related to a higher risk perception among MSM. Previous study has reported higher perceived HIV risk was associated with greater willingness to use PrEP (32). In Nigeria, MSM who engaged in oral sex have reported high prevalence of oropharyngeal STDs (33). Similarly, a study conducted by Templeton et al. (34) revealed that MSM who often engaged in insertive oro-anal sex were more likely to have oropharyngeal gonorrhea than MSM who never engaged. Again, Cornelisse et al. (35)also found that the majority of MSM with urethral gonorrhea 
had acquired their infection from the rectum or pharynx of their partner. Moreover, having STDs increased the risk of HIV acquisition.

Results of this study showed that participants who had sex with casual man in the past six months were associated to lower willingness to use PrEP. Further studies are needed to understand the association. This study suggest that PrEP might be perceived as less important for MSM of ever had casual sex. Having sex with casual male partner might increase HIV acquisition risk; because it was unplanned sexual encounter, and it might be challenging to know partner sero-status. HIV sero-status disclosure might serve as a way to reduce HIV acquisition and transmission risk and it might help MSM make informed decisions about safe sexual practice (36). In previous study, condom less anal sexual intercourse was increased with casual partner in the past three months (37). Therefore, this population was under high HIV acquisition risk and it was necessary to identify if PrEP-related knowledge, awareness and stigma described in similar studies $(38,39)$ could be related to this perception.

On the contrary, MSM who had taken up HIV testing in the past showed willingness to use PrEP and which was similar to study conducted in Hong Kong (40). Since $68 \%$ of our participants had taken up HIV testing in past, it was possible that MSM might have been counselled on the efficacy of PrEP to decrease HIV transmission among them. HIV counselling and testing was the first step in making MSM aware of their sero-status. It was also important to facilitate early access to treatment; enabling the success of biomedical interventions including treatment as prevention (41). Study by Werner et al. (29)revealed that $14 \%$ MSM got knowledge about PrEP from counseling centers. Similarly, in US, overall uptake of PrEP was high when offered in sexual transmitted diseases clinics and a community health center (42). These facilities might be an ideal setting to promote PrEP. In summary, this study provided important implications for the implementation of PrEP by combining with other behavioral approaches for HIV prevention among MSM. This study had several limitations. 1. Participants were recruited only from Liuzhou area; so results might not be generalizable to all MSM in China. 2. Sexual behaviors relied on participants self-report and might be subject to recall bias. 3 . The reasons for unwillingness to use PrEP were not assessed. Despite these limitations, this was the first quantitative analyses of PrEP willingness and associated factors among MSM in Liuzhou, China. Additionally, this was the first study, which was conducted in HIV-negative MSM at the study area. Furthermore, the participants of our study were recruited from several sampling sources, which provided for a diverse sample of varied experiences.

\section{Conclusions}

These findings indicate that willingness to use PrEP among MSM remains low, however, many of them engaged in high-risk behavior. Therefore, there is an urgent need to increase MSM's awareness of PrEP and increase their knowledge about HIV prevention. In addition, Chinese public health authorities should prioritize the implementation of PrEP, which may reduce new HIV infection cases in MSM and HIV related medical costs.

\section{List Of Abbreviations}


PrEP: Pre-exposure prophylaxis; HIV: Human immunodeficiency virus; AIDS: Acquired immune deficiency syndrome; MSM: Men who have sex with men; US: United States; STIs: Sexually transmitted infections; ART: Antiretroviral therapy; WHO: World Health Organization; CDC: Centers for Disease Control and Prevention; STDs: Sexually transmitted diseases; SD: standard deviation; ORs: Odd ratios; CI: Confidence intervals; IQR: Interquartile range; aOR: Adjusted odd ratio

\section{Declarations}

\section{Ethics approval and consent to participate}

This study was approved by the ethics committee of Anhui Medical University. Written informed consent was obtained from each subject prior to enrolling in this study.

\section{Consent for publication}

Not applicable.

\section{Availability of data and materials}

The datasets used and/or analyzed during the current study are available from the corresponding author on reasonable request.

\section{Competing interests}

The authors declare that they have no competing interests.

\section{Funding}

This project was supported by the program of technical support for Liuzhou city (K2020043), which was authorized by the Chinese National Center for AIDS/STD Control and Prevention.

\section{Authors' contributions}

YF and DY contributed to the conception and design of the study. YF, XF, TW and SZ collected the data. YF and AAA analyzed the data. YF and AAA drafted the initial manuscript. All the authors have read and approved the final manuscript.

\section{Acknowledgements}

We appreciate all the participants who volunteered to participate in this study.

\section{References}

1. GBD 2015 HIV Collaborators. Estimates of global, regional, and national incidence, prevalence, and mortality of HIV, 1980-2015: the Global Burden of Disease Study 2015. Lancet HIV. 2016;3(8):e361- 
e87.

2. Chinese Center for Disease Control and Prevention. Update on the AIDS /STD epidemic in China the third quarter of 2018. Chin J AIDS STD. 2018;24(11):1075.

3. Wu Z. Achievement of HIV/AIDS program in the past 30 years and challenges in China. Chin $\mathrm{J}$ Epidemiol. 2015;36(12):1329-31.

4. Peng P, Su S, Fairley CK, Chu M, Jiang S, Zhuang X, et al. A Global Estimate of the Acceptability of Pre-exposure Prophylaxis for HIV Among Men Who have Sex with Men: A Systematic Review and Meta-analysis. AIDS Behav. 2018;22(4):1063-74.

5. Zhenhua D, Shuangfeng F, Rong L, Xueqing W, Yaying S, Zhijun L, et al. Consistently high HIV prevalence among men who have sex with men in Chengdu city from 2009 to 2014. Int J STD AIDS. 2016;27(12):1057-62.

6. Zeng G, Feng L, Ouyang L, Lu R, Xu P, Wu G, et al. The dynamic trends of HIV prevalence, risks, and prevention among men who have sex with men in Chongqing, China. Biomed Res Int. 2014;2014:602719.

7. WHO Guidelines Approved by the Guidelines Review Committee. Consolidated Guidelines on HIV Prevention, Diagnosis, Treatment and Care for Key Populations: World Health Organization: Geneva; 2014.

8. Grant RM, Anderson PL, McMahan V, Liu A, Amico KR, Mehrotra M, et al. Uptake of pre-exposure prophylaxis, sexual practices, and HIV incidence in men and transgender women who have sex with men: a cohort study. Lancet Infect Dis. 2014;14(9):820-9.

9. Baeten JM, Donnell D, Ndase P, Mugo NR, Campbell JD, Wangisi J, et al. Antiretroviral prophylaxis for HIV prevention in heterosexual men and women. N Engl J Med. 2012;367(5):399-410.

10. Choopanya K, Martin M, Suntharasamai P, Sangkum U, Mock PA, Leethochawalit M, et al. Antiretroviral prophylaxis for HIV infection in injecting drug users in Bangkok, Thailand (the Bangkok Tenofovir Study): a randomised, double-blind, placebo-controlled phase 3 trial. Lancet. 2013;381(9883):2083-90.

11. WHO Guidelines Approved by the Guidelines Review Committee. Guideline on When to Start Antiretroviral Therapy and on Pre-Exposure Prophylaxis. for: World Health Organization: Geneva;; 2015.

12. Dong M-J, Peng B, Liu Z-F, Ye Q-N, Liu H, Lu X-L, et al. The prevalence of HIV among MSM in China: a large-scale systematic analysis. BMC Infect Dis. 2019;19(1):1000.

13. Zeng $X$, Zhong X, Peng B, Zhang Y, Kong C, Liu X, et al. Prevalence and associated risk characteristics of HIV infection based on anal sexual role among men who have sex with men: a multi-city crosssectional study in Western China. Int J Infect Dis. 2016;49:111-8.

14. Hoagland B, De Boni RB, Moreira RI, Madruga JV, Kallas EG, Goulart SP, et al. Awareness and Willingness to Use Pre-exposure Prophylaxis (PrEP) Among Men Who Have Sex with Men and Transgender Women in Brazil. AIDS Behav. 2017;21(5):1278-87. 
15. Sullivan PS, Sanchez TH, Zlotorzynska M, Chandler CJ, Sineath RC, Kahle E, et al. National trends in HIV pre-exposure prophylaxis awareness, willingness and use among United States men who have sex with men recruited online, 2013 through 2017. J Int AIDS Soc. 2020;23(3):e25461.

16. Wang Z, Lau JTF, Fang Y, Ip M, Gross DL. Prevalence of actual uptake and willingness to use preexposure prophylaxis to prevent HIV acquisition among men who have sex with men in Hong Kong, China. PLoS ONE. 2018;13(2):e0191671.

17. Zhang Y-J, Feng X-X, Fan Y-G, Jiang Z-Y, Zhong X-H, Li M-Q, et al. HIV transmission and related risk factors among serodiscordant couples in Liuzhou, China. J Med Virol. 2015;87(4):553-6.

18. Wei I, Feng W-D, Fan Y-G, Shan G-S. Analysis of AIDS epidemic in Liuzhou City of Guangxi Province in 2015. J Med Pest Control. 2017;33(3):255-7.

19. Ding Y, Yan H, Ning Z, Cai X, Yang Y, Pan R, et al. Low willingness and actual uptake of pre-exposure prophylaxis for HIV-1 prevention among men who have sex with men in Shanghai, China. Biosci Trends. 2016;10(2):113-9.

20. Peng L, Cao W, Gu J, Hao C, Li J, Wei D, et al. Willingness to Use and Adhere to HIV Pre-Exposure Prophylaxis (PrEP) among Men Who Have Sex with Men (MSM) in China. Int J Environ Res Public Health. 2019;16(14).

21. Grov C, Whitfield THF, Rendina HJ, Ventuneac A, Parsons JT. Willingness to Take PrEP and Potential for Risk Compensation Among Highly Sexually Active Gay and Bisexual Men. AIDS Behav. 2015;19(12):2234-44.

22. Hoots BE, Finlayson T, Nerlander L, Paz-Bailey G. Willingness to Take, Use of, and Indications for Preexposure Prophylaxis Among Men Who Have Sex With Men-20 US Cities, 2014. Clin Infect Dis. 2016;63(5):672-7.

23. Young I, Li J, McDaid L. Awareness and willingness to use HIV pre-exposure prophylaxis amongst gay and bisexual men in Scotland: implications for biomedical HIV prevention. PLoS ONE. 2013;8(5):e64038.

24. Ferrer L, Folch C, Fernandez-Davila P, Garcia A, Morales A, Belda J, et al. Awareness of Pre-exposure Prophylaxis for HIV, Willingness to Use It and Potential Barriers or Facilitators to Uptake Among Men Who Have Sex with Men in Spain. AIDS Behav. 2016;20(7):1423-33.

25. Garofalo R, Gayles T, Bottone PD, Ryan D, Kuhns LM, Mustanski B. Racial/Ethnic Difference in HIVrelated Knowledge among Young Men who have Sex with Men and their Association with Condom Errors. Health Educ J. 2015;74(5):518-30.

26. Magno L, Dourado I, Sutten Coats C, Wilhite D, da Silva LAV, Oni-Orisan O, et al. Knowledge and willingness to use pre-exposure prophylaxis among men who have sex with men in Northeastern Brazil. Glob Public Health. 2019;14(8):1098-111.

27. Khosravi N, Kolifarhood G, Shoghli A, Pashaeypoor S, Amlashi M. Effectiveness of peer education approach on improving HIV/AIDS related healthy behaviors among immigrant street children: A randomized controlled trial. Clinical Epidemiology Global Health. 2018;6(3):115-21. 
28. Shangani S, Escudero D, Kirwa K, Harrison A, Marshall B, Operario D. Effectiveness of peer-led interventions to increase HIV testing among men who have sex with men: a systematic review and meta-analysis. AIDS Care. 2017;29(8):1003-13.

29. Werner RN, Gaskins M, Ahrens J, Jessen H, Kutscha F, Mosdzen R, et al. Knowledge and use of HIV pre-exposure prophylaxis among men who have sex with men in Berlin - A multicentre, crosssectional survey. PLoS ONE. 2018;13(9):e0204067.

30. Lau JTF, Zhao J-K, Wu X-B, Gu J, Hao C. Gay saunas and the risks of HIV and syphilis transmissions in China-results of a meta-analysis. J Sex Med. 2013;10(3):642-52.

31. Bonell C, Strange V, Allen E, Barnett-Page E. HIV prevention outreach in commercial gay venues in large cities: evaluation findings from London. Health Educ Res. 2006;21(4):452-64.

32. Stein M, Thurmond P, Bailey G. Willingness to use HIV pre-exposure prophylaxis among opiate users. AIDS Behav. 2014;18(9):1694-700.

33. Robbins SJ, Dauda W, Kokogho A, Ndembi N, Mitchell A, Adebajo S, et al. Oral sex practices among men who have sex with men and transgender women at risk for and living with HIV in Nigeria. PLoS ONE. 2020;15(9):e0238745.

34. Templeton DJ, Jin F, McNally LP, Imrie JCG, Prestage GP, Donovan B, et al. Prevalence, incidence and risk factors for pharyngeal gonorrhoea in a community-based HIV-negative cohort of homosexual men in Sydney, Australia. Sex Transm Infect. 2010;86(2):90-6.

35. Cornelisse VJ, Zhang L, Law M, Chen MY, Bradshaw CS, Bellhouse C, et al. Concordance of gonorrhoea of the rectum, pharynx and urethra in same-sex male partnerships attending a sexual health service in Melbourne, Australia. BMC Infect Dis. 2018;18(1):95.

36. Grov C, Agyemang L, Ventuneac A, Breslow AS. Navigating condom use and HIV status disclosure with partners met online: a qualitative pilot study with gay and bisexual men from Craigslist.org. AIDS Educ Prev. 2013;25(1):72-85.

37. Hoornenborg E, Coyer L, Achterbergh RCA, Matser A, Schim van der Loeff MF, Boyd A, et al. Sexual behaviour and incidence of HIV and sexually transmitted infections among men who have sex with men using daily and event-driven pre-exposure prophylaxis in AMPrEP: 2 year results from a demonstration study. Lancet HIV. 2019;6(7):e447-e55.

38. Zhou F, Gao L, Li S, Li D, Zhang L, Fan W, et al. Willingness to accept HIV pre-exposure prophylaxis among Chinese men who have sex with men. PLoS ONE. 2012;7(3):e32329.

39. Newman PA, Guta A, Lacombe-Duncan A, Tepjan S. Clinical exigencies, psychosocial realities: negotiating HIV pre-exposure prophylaxis beyond the cascade among gay, bisexual and other men who have sex with men in Canada. J Int AIDS Soc. 2018;21(11):e25211.

40. Wang Z, Mo PKH, Ip M, Fang Y, Lau JTF. Uptake and willingness to use PrEP among Chinese gay, bisexual and other men who have sex with men with experience of sexualized drug use in the past year. BMC Infect Dis. 2020;20(1):299.

41. Dalrymple J, McAloney-Kocaman K, Flowers P, McDaid LM, Frankis JS. Age-related factors influence HIV testing within subpopulations: a cross-sectional survey of MSM within the Celtic nations. Sex 
Transm Infect. 2019;95(5):351-7.

42. Patrick R, Forrest D, Cardenas G, Opoku J, Magnus M, Phillips G, et al. Awareness, Willingness, and Use of Pre-exposure Prophylaxis Among Men Who Have Sex With Men in Washington, DC and Miami-Dade County, FL: National HIV Behavioral Surveillance, 2011 and 2014. J Acquir Immune Defic Syndr. 2017;75(Suppl 3):375-S82.

\section{Tables}

Table 1 Background characteristics and willingness to use PrEP 


\begin{tabular}{|c|c|c|c|c|c|}
\hline Variables & $\begin{array}{c}\text { Total } \\
(\mathrm{N}=829) \\
\mathrm{n}(\%)\end{array}$ & $\begin{array}{l}\text { Unwilling to use PrEP } \\
(\mathrm{n}=578) \mathrm{n}(\%)\end{array}$ & $\begin{array}{c}\text { Willing } \\
\text { to } \\
\text { use } \\
\text { PrEP } \\
(\mathrm{n}=251) \\
\mathrm{n}(\%) \\
\end{array}$ & $c^{2} / t$ & $P$ value \\
\hline Age group (years) & \multirow{5}{*}{$\begin{array}{c}342 \\
(41.3) \\
323 \\
(39.0) \\
164 \\
(19.8)\end{array}$} & & \multirow{5}{*}{$\begin{array}{c}100 \\
(39.8) \\
95(37.8) \\
56(22.3)\end{array}$} & 1.452 & 0.484 \\
\hline $15-24$ & & $242(49.1)$ & & & \\
\hline $25-34$ & & $228(39.4)$ & & & \\
\hline$\geq 35$ & & $108(18.7)$ & & & \\
\hline Household registration & & & & 2.920 & 0.087 \\
\hline Local (Liuzhou) & \multirow{3}{*}{$\begin{array}{c}475 \\
(57.3) \\
354 \\
(42.7)\end{array}$} & $320(55.4)$ & \multirow{3}{*}{$\begin{array}{c}155 \\
(61.8) \\
96(38.2)\end{array}$} & & \\
\hline Non-local & & $258(44.6)$ & & & \\
\hline Ethnicity & & & & 46.581 & $<0.001$ \\
\hline Zhuang & \multirow{4}{*}{$\begin{array}{c}315 \\
(38.0) \\
362 \\
(43.7) \\
152 \\
(18.3)\end{array}$} & $238(41.2)$ & $77(30.7)$ & & \\
\hline Han & & $210(36.3)$ & 152 & & \\
\hline Others & & $130(22.5)$ & $22(8.8)$ & & \\
\hline Occupation & & & & 3.974 & 0.410 \\
\hline Students & \multirow{6}{*}{$\begin{array}{c}159 \\
(19.2) \\
155 \\
(18.7) \\
167 \\
(20.1) \\
201 \\
(24.2) \\
147 \\
(17.7)\end{array}$} & $109(18.9)$ & $50(19.9)$ & & \\
\hline $\begin{array}{l}\text { Company employees } \\
\text { /government staff }\end{array}$ & & $117(20.2)$ & $38(15.1)$ & & \\
\hline Factory workers & & 115 (19.9) & $52(20.7)$ & & \\
\hline Freelancers & & $141(24.4)$ & $60(23.9)$ & & \\
\hline Others & & $96(16.6)$ & $51(20.3)$ & & \\
\hline Education level & & & & 6.323 & 0.042 \\
\hline Middle school or below & \multirow{4}{*}{$\begin{array}{c}123 \\
(14.8) \\
129 \\
(15.6) \\
577 \\
(69.6)\end{array}$} & $96(16.6)$ & $27(10.8)$ & & \\
\hline High school & & $94(16.3)$ & $35(13.9)$ & & \\
\hline College or above & & $388(67.1)$ & $\begin{array}{c}189 \\
(75.3)\end{array}$ & & \\
\hline Marital status & & & & 3.430 & 0.180 \\
\hline Never married & \multirow{4}{*}{$\begin{array}{c}603 \\
(72.7) \\
175 \\
(21.1) \\
51(6.2)\end{array}$} & $428(74.0)$ & $\begin{array}{c}175 \\
(697)\end{array}$ & & \\
\hline Married & & $120(20.8)$ & $55(21.9)$ & & \\
\hline Divorced / widowed & & $30(5.2)$ & $21(8.4)$ & & \\
\hline $\begin{array}{l}\text { Personal monthly } \\
\text { income(RMB) }\end{array}$ & & & & & \multirow{5}{*}{0.027} \\
\hline$<1500$ & \multirow{5}{*}{$\begin{array}{c}137 \\
(16.5) \\
195 \\
(23.5) \\
347 \\
(41.9) \\
150 \\
(18.1)\end{array}$} & $110(19.0)$ & $27(10.8)$ & \multirow[t]{4}{*}{9.193} & \\
\hline $1500-3000$ & & $134(23.2)$ & $61(24.3)$ & & \\
\hline $3001-4500$ & & $236(40.8)$ & $\begin{array}{c}111 \\
(44.2)\end{array}$ & & \\
\hline$>4500$ & & $98(17.0)$ & $52(20.7)$ & & \\
\hline Sexual orientation & & & & 9.498 & 0.002 \\
\hline Homosexual & 632 & $458(79.2)$ & 174 & & \\
\hline
\end{tabular}


Table 2 Sexual behavior characteristics and willingness to use PrEP. 


\begin{tabular}{cccccc}
\hline Varibles & Total & Unwilling to use & Willing to \\
$(\mathrm{N}=829)$ & $\mathrm{PrEP}(\mathrm{n}=578) \mathrm{n}$ & use PrEP \\
$\mathrm{n}(\%)$ & $(\mathrm{n}=251) \mathrm{n}$ & $c^{2} / t$ & $P$ value \\
& & & & \\
& & & &
\end{tabular}

Number of male sex partners in lifetime

$$
1
$$

$2-5$

6-10

11-20

$>20$

Ever had anal sex with a man

Yes

No

Age of first anal sex with a 22.64 26.34 man (years)

Number of male anal sex partners in the past month

$\leq 1$
$2-5$
$6-10$
$>10$

Condom use at last anal sex with a man

Yes

No

Ever had oral sex with a man

Yes

No

Number of male oral sex partners in the past month

$\leq 1$
$2-5$
$6-10$
$>10$

Had commercial sex in the past six months

Yes

No

Had casual sex other than commercial sex in the past six months

Yes

No

Number of female sex partners in lifetime

0

1

$2-5$

$774(93.4)$

$55(6.6)$

$561(72.5)$

$213(27.5)$

$738(89.0)$

91 (11.0)

$42(5.1)$

787 (94.9)

$\begin{array}{ccc}82(9.9) & 64(11.1) & 18(7.2) \\ 404(48.7) & 287(49.7) & 117(46.6) \\ 157(18.9) & 102(17.6) & 55(21.9) \\ 88(10.6) & 60(10.4) & 28(11.2) \\ 98(11.8) & 65(11.2) & 33(13.1)\end{array}$

544 (94.1)

34 (5.9)

$22.57 \pm 6.62$

$412(53.2)$
$309(39.9)$
$33(4.3)$
$20(2.6)$

287 (52.8)

222 (40.8)

21 (3.9)

14 (2.6)

385 (70.8)

159 (29.2)

502 (86.9)

76 (13.1)
355 (55.4)
245 (38.2)
$28(4.4)$
$13(2.0)$
246 (58.4)
167 (38.3)
$18(4.1)$
$5(1.1)$

$31(5.4)$

547 (94.6)

11 (4.4)

240 (95.6)

109 (53.2)

78 (38.0)

$10(4.9)$

8 (3.9)

$2.679 \quad 0.102$

$176(76.5)$

54 (23.5)

$236(94.0)$

$15(6.0)$

$9.213 \quad 0.002$

$5.669 \quad 0.129$

$0.350 \quad 0.554$

$19.179<0.001$

$\begin{array}{llc}335(40.4) & 262(45.3) & 73(29.1) \\ 494(59.6) & 316(54.7) & 178(70.9)\end{array}$

$3.849 \quad 0.427$

$456(55.0) \quad 323(55.9) \quad 133(53.0)$

$227(27.4) \quad 163(28.2) \quad 64(25.5)$

$127(15.3) \quad 80(13.8) \quad 47(18.7)$ 


\begin{tabular}{|c|c|c|c|c|c|}
\hline $\begin{array}{l}6-10 \\
>10\end{array}$ & $\begin{array}{l}11(1.3) \\
8(1.0)\end{array}$ & $\begin{array}{l}7(1.2) \\
5(0.9)\end{array}$ & $\begin{array}{l}4(1.6) \\
3(1.2)\end{array}$ & & \\
\hline Age of first sex with a & $22.15 \pm 3.90$ & $22.16 \pm 3.92$ & $22.12 \pm 3.86$ & 0.085 & 0.933 \\
\hline 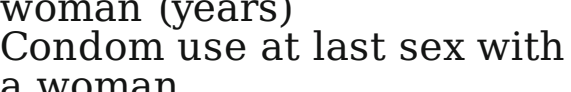 & & & & 0.472 & 0.492 \\
\hline $\begin{array}{l}\text { A woman } \\
\text { Yes }\end{array}$ & $145(43.4)$ & $93(42.1)$ & $52(46.0)$ & & \\
\hline No & $189(56.6)$ & $128(57.9)$ & $61(54.0)$ & & \\
\hline
\end{tabular}

Table 3 Logistic regression analysis of factors associated with willingness to use PrEP. 


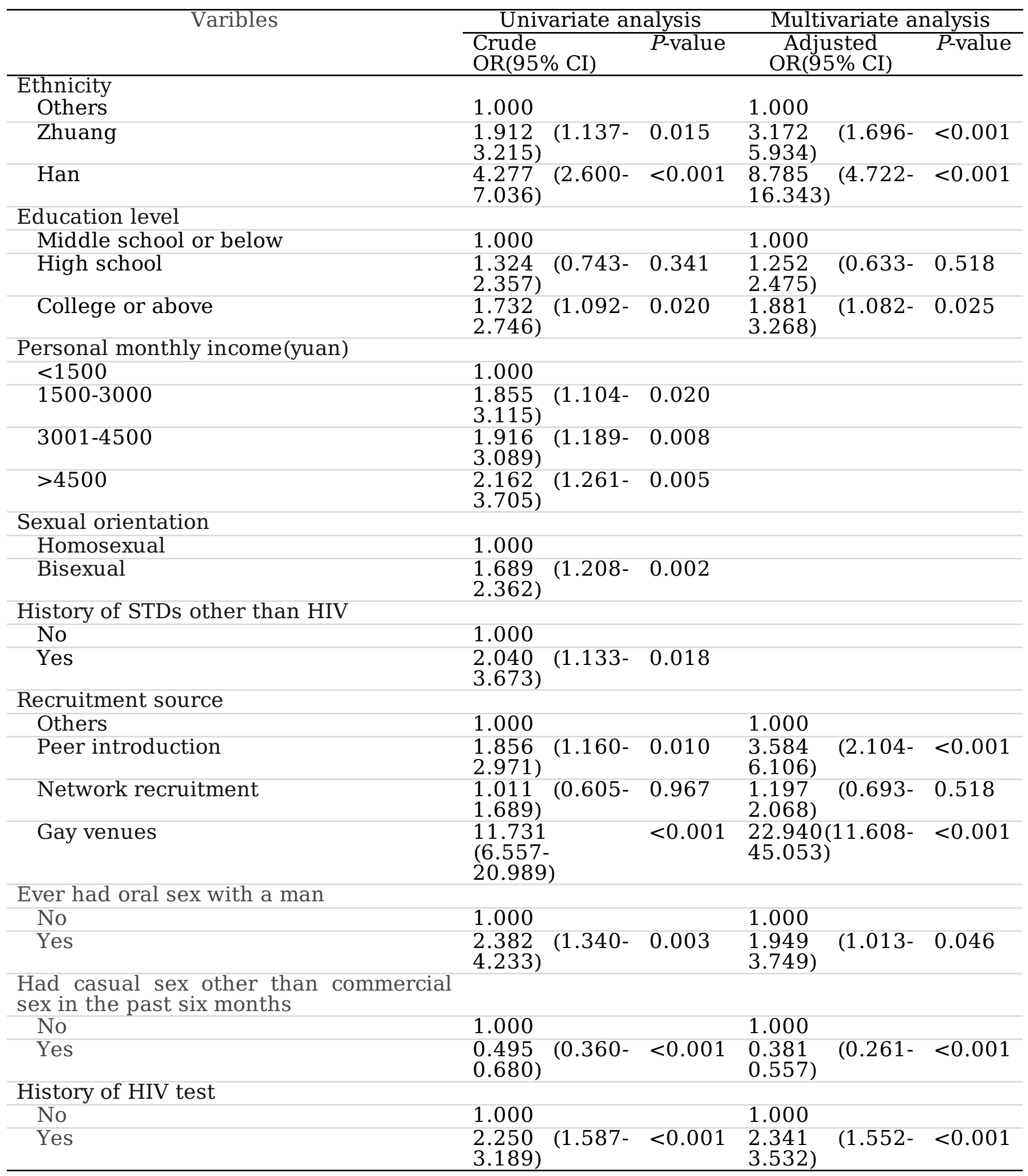

